\title{
Valacyclovir Hydrochloride
}

National Cancer Institute

\section{Source}

National Cancer Institute. Valacyclovir Hydrochloride. NCI Thesaurus. Code C29535.

The hydrochloride salt of valacyclovir. Valacyclovir is an acyclovir prodrug that, after metabolization, inhibits viral DNA replication. It is used in the management of herpes simplex and varicella zoster infections, as well as prophylactically for human cytomegalovirus infections. 